\title{
La Ingeniería de Ecosistemas
}

\section{Ecosystems Engineering}

\author{
Ernesto Guhl Nannetti ${ }^{(1)}$ \\ (1) Ingeniero civil. Profesor, ex decano de ingeniería y ex vicerrector de la Universidad de los Andes, Presidente de la Asociación Colom- \\ biana de Facultades de Ingeniería - ACOFI, Ex viceministro del medio ambiente y Director del Instituto Quinaxi. Bogotá, Colombia. \\ eguhl@quinaxi.org
}

Recibido 15 de julio de 2015. Modificado 23 de julio de 2015. Aprobado 24 de julio de 2015.

DOI: http://dx.doi.org/10.16924/ riua.v0i42.829

\section{Palabras clave}

Ambiente, antropoceno, conservación, desarrollo, ecosistema, impacto ambiental, ingeniería, programa, sostenibilidad, territorio

\section{Resumen}

Este artículo inicia tratando la evolución de la ingeniería con el avance de las ciencias físicas y naturales y la especialización resultante, lo que ha desdibujado su naturaleza integradora y holística. Se ilustra este proceso con la expansión de los programas y las denominaciones en Colombia. Más adelante, trata la relación entre la ingeniería y la sostenibilidad y, presenta el alto impacto ambiental de las formas de desarrollo que ha llevado a denominar este período de la historia como el Antropoceno. Finalmente, se propone un enfoque de la ingeniería buscando que contribuya a la conservación y al fortalecimiento de los ecosistemas, como proveedores de servicios vitales que son la base del progreso, ayudando a la generación de territorios sostenibles. Esta propuesta, compleja e integradora, se denomina como Ingeniería de Ecosistemas.

\section{Key words}

Anthropocene, environment, conservation, development, ecosystem, engineering, environment, environmental impact, program, sustainability, territory.

\begin{abstract}
The article begins by referring to the evolution of engineering with the advancement of natural and physical sciences and the resulting specialization, which has blurred the boundaries of its integrating and holistic nature. This process is illustrated through the expansion of the programs and denominations in Colombia. Further on, it attempts to establish a relationship between engineering and sustainability, and it presents the strong impact of development on the planet's ecosystems, which has led this era to be known as the Anthropocenic period. Finally, we propose a new approach for engineering in which it can contribute to the conservation and development of the ecosystems as providers of vital services needed for progress and social advancement and to the fostering of sustainable territories. This new holistic and complex approach to engineering is known as Ecosystems Engineering.
\end{abstract}

\section{CONCEPCIÓN Y EVOLUCIÓN DE LA INGENIERÍA}

Pueden darse muchas definiciones de la ingeniería ya que, ésta varía a lo largo del tiempo, en la medida en que incorpora nuevos temas y problemas y nuevos métodos y herramientas para tratarlos. Sin embargo, en términos generales la ingeniería puede entenderse como el conjunto de conocimientos, técnicas y herramientas que permiten utilizar y transformar los recursos naturales y el paisaje, para satisfacer las necesidades y las expectativas de progreso de la sociedad.

Así pues, la ingeniería es una disciplina integradora y dinámica que se basa fundamentalmente en la aplicación de las leyes de las ciencias físicas y químicas, el apoyo de herramientas matemáticas y estadísticas y en visiones, conocimientos y métodos provenientes de las ciencias sociales y naturales. El proceso evolutivo que sigue la ingeniería a lo largo del tiempo va desarrollándose en la medida en que los avances científicos ponen a su disposición nuevos campos de acción y los intereses y las necesidades de las sociedades van cambiando y haciendo nuevas exigencias para satisfacerlos.

Es indispensable que la ingeniería, como ciencia aplicada, tenga una sólida base teórica en las ciencias físicas, químicas y matemáticas, que permita a los ingenieros contar con los conocimientos teóricos para resolver de manera creativa, eficaz y eficiente los 
problemas que se le plantean en su actividad profesional. Al decir de Jorge Luis Borges, la realidad es hija de la teoría, por lo cual la generación de conocimiento en las ciencias básicas mediante la investigación es un requisito indispensable para abordar los problemas, desarrollando productos y proyectos que respondan de la mejor manera posible a las características del entorno, a las necesidades sociales y a las disponibilidades de recursos humanos, tecnológicos y financieros.

Sin entrar en detalle, por razones de espacio, en la importancia de los avances tecnológicos iniciales del "homo sapiens” que le han permitido convertirse paulatinamente en la especie dominante del planeta, como el uso del fuego, el manejo y el uso de la piedra, la madera y los metales, ya para el siglo XVII se crean las primeras escuelas profesionales de ingeniería en Francia, Alemania y otros países europeos. A partir de esa época y respondiendo a su carácter evolutivo y específico, la ingeniería ha ido ampliándose desde la Ingeniería Militar empleada para la guerra, a la Ingeniería Civil, llamada así para diferenciarla de la anterior, la cual abarcaba un conjunto muy amplio de actividades como el diseño y la construcción de edificios civiles y religiosos, caminos, puertos, acueductos, barcos, entre otras.

En la medida en que fueron desarrollándose las diversas ciencias y disciplinas como la hidráulica, la mecánica, las estructuras, la minería, la electricidad y el magnetismo, las fuentes de energía, la agronomía, los transportes, la genética, y demás... fueron conformándose también especialidades de la ingeniería en los distintos campos para satisfacer las necesidades básicas y culturales de la sociedad.

Sin embargo, en este proceso se ha perdido la visión holística y la integralidad original de la ingeniería, especializando cada vez más sus programas profesionales, con lo cual el carácter del ingeniero ha cambiado pasando de ser un profesional capaz de abordar diferentes problemas a convertirse en especialista en un determinado campo de la profesión. De acuerdo con la Asociación Colombiana de Facultades de Ingeniería, ACOFI, el número de programas de pregrado en ingeniería pasó de 200 en 1992 a 886 en 2012. Según el Sistema Nacional de Información de la Educación Superior, SNIES (consultado por la ACOFI el 21 de mayo de 2014), existían en el país 1305 programas de pregrado, de los cuales 913 estaban activos, que se distribuían en 89 titulaciones o denominaciones, de las cuales 49 cuentan con uno o más programas en ingeniería acreditados y de ellos apenas el 25\% contaba con reconocimiento de alta calidad.

En cuanto al número, la cifra ya se acerca al millón de estudiantes de ingeniería, que corresponde al $27 \%$ de la matrícula total de pregrado en el país. De ellos, el $71 \%$ se concentraba en 6 ingenierías; Civil, Sistemas y afines, Industrial, Mecánica, Electrónica y Ambiental. El resto se distribuía en 70 titulaciones diferentes con el nombre de ingeniería. Como puede apreciarse, el deseo de especialización y el atractivo económico de ofrecer nuevos programas ha conducido a una explosión en la cantidad y la especialidad de programas de ingeniería que muchas veces carecen de los fundamentos teóricos necesarios, cuyos egresados corresponden mejor al perfil de tecnólogos. En relación con los programas de posgrado en ingeniería, en 2012 existían en el país alrededor de 700 especializaciones, 200 maestrías y 38 doctorados de acuerdo con la ACOFI.

\section{EL IMPACTO HUMANO Y LOS LÍMITES DE LA NATURALEZA. EL ANTROPOCENO}

Con respecto a este tema es necesario destacar que, en términos generales, la cultura occidental entiende la naturaleza, aún hoy lamentablemente, como una fuente inagotable de recursos puestos a disposición del ser humano. Estos recursos se han clasificado en renovables y no renovables, categorías que corresponden esencialmente a la separación entre los recursos minerales y los biológicos. Los primeros existen en cantidades importantes pero finitas en el planeta, pero los segundos dada su naturaleza y su capacidad de reproducción y regeneración, se consideran inagotables. En esta última categoría se ha incluido muy equivocadamente al agua.

Hoy en día, para nadie es desconocido que los efectos de la cada vez más fuerte e invasiva intervención humana sobre los ecosistemas planetarios los está afectando severamente. Estos impactos son tan cumulativos y fuertes, en especial a partir de la segunda mitad del siglo pasado que este período se ha denominado como el Antropoceno, aludiendo a que el ser humano se ha convertido en un poderoso factor de cambio de las condiciones y las características ambientales del planeta. El fenómeno de la destrucción del mundo natural y la artificialización del planeta están generando una serie de transformaciones en diversos campos que se han denominado como Cambio Global.

Desde el punto de vista del efecto sobre la biodiversidad, este impacto ha sido tan fuerte que estamos viviendo una acelerada desaparición de especies, tan severa que se ha denominado como la Sexta Extinción Masiva. Si bien es cierto que ha habido cinco extinciones masivas anteriores en la historia del planeta, la diferencia fundamental de la actual con las anteriores, es que ésta es causada por las actividades humanas. En su último libro La Sexta Extinción- Una historia nada natural, Elizabeth Kolbert, estima que al final del siglo XXI, es decir en alrededor de 80 años, habrán desaparecido entre el 20\% y el 50\% de todas las especies vivas que existían sobre la Tierra. No tenemos idea clara de cuáles serán las implicaciones de esta desaparición sobre los ecosistemas, su funcionamiento y la generación de sus bienes y servicios que son indispensables para la vida y el progreso social, dado que los ecosistemas son complejas redes de vida en la que los diversos seres vivos desarrollan funciones interdependientes que 
contribuyen al todo. Esta desaparición masiva de nuestros compañeros de casa planetaria tiene unos efectos desconocidos e inciertos para el futuro de la humanidad.

Para ilustrar el problema anterior en la Figura 1 se presenta el Índice Planeta Vivo que nos muestra cómo las poblaciones de vertebrados disminuyeron casi el $30 \%$ entre 1970 y 2007.

En lo que respecta a la consideración de la Huella Ecológica como indicadora de la insostenibilidad del modelo económico vigente, el capitalismo global desregulado, la Figura 2 muestra la cantidad de planetas Tierra necesarios para satisfacer nuestras necesidades como sociedad con los niveles de consumo vigentes. Como puede observarse, alrededor de 1975, copamos la biocapacidad del planeta y en la primera década del presente siglo llegamos a utilizar más de 1.5 Tierras. Es decir que por primera vez en la historia humana hemos sobrepasado los límites de la naturaleza y estamos afectando el funcionamiento del planeta. Naturalmente esta tendencia implica un deterioro de la capacidad de los ecosistemas planetarios para mantener el crecimiento y el nivel de consumo que implica el sistema económico actual, poniendo de presente con toda claridad su insostenibilidad.

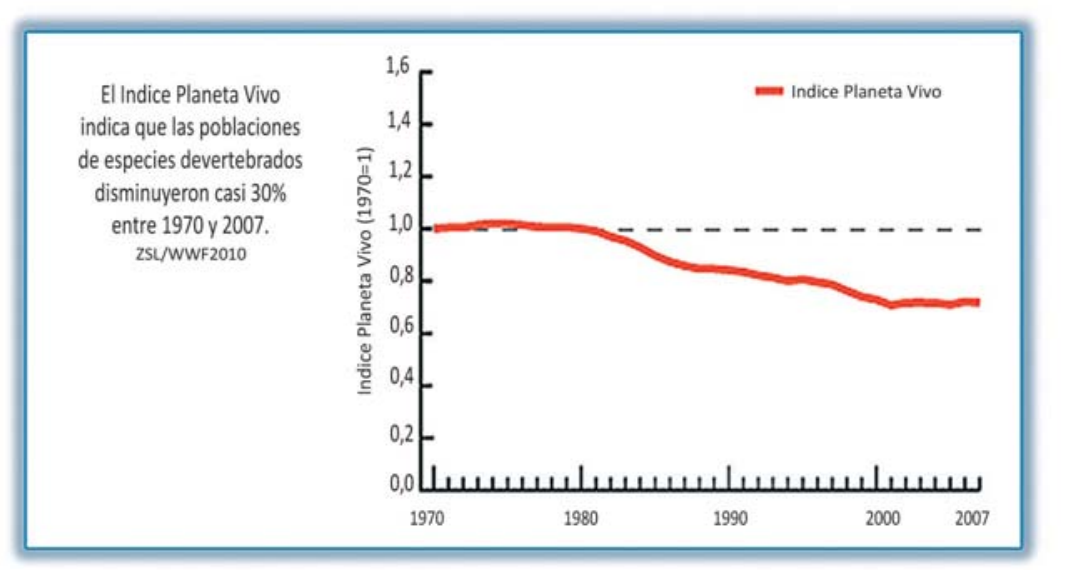

Figura 1. Índice de Planeta Vivo. Fuente: WWF et al., 2010.

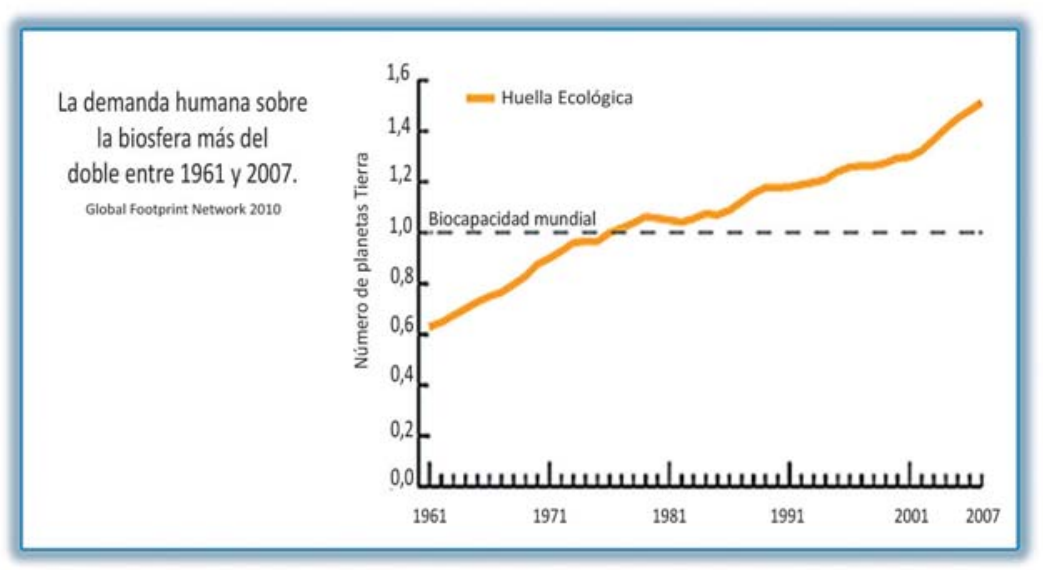

Figura 2. Huella ecológica mundial. Fuente: Global Footprint Network.
Tal vez el ejemplo más conocido y grave del Cambio Global es el cambio climático. Las emisiones de gases de efecto invernadero GEI, derivados de la quema de combustibles fósiles, la deforestación y la ganadería principalmente, están modificando aceleradamente la composición química de la atmósfera, induciendo un aumento de la temperatura que se manifiesta en la pérdida de los casquetes glaciales, el aumento del nivel del mar, la acidificación de los mares, fuertes alteraciones en los patrones climáticos "normales", cambios imprevistos en la distribución e intensidad de las lluvias, el aumento de la frecuencia de los fenómenos hidrometeorológicos extremos, como inundaciones y sequías y la desaparición o disminución de la extensión de ecosistemas esenciales como los páramos.

En realidad el cambio climático es la amenaza más grande que ha tenido la especie humana a lo largo de toda su historia. Casi todos los escenarios que se han planteado como resultado de los efectos de diversos aumentos de la temperatura, prevén efectos catastróficos, cuyos costos de remediación son equivalentes a varios puntos del PIB mundial. Adicionalmente, en estos escenarios están siempre presentes la incertidumbre y el temor de que si la temperatura sobrepasa lo que se considera un límite "seguro" de incremento, que se estima en $2^{\circ} \mathrm{C}$, por encima del nivel anterior a que se empezara a utilizar el carbono masivamente como combustible, se pueden generar puntos de inflexión no lineales y saltos irreversibles en las condiciones ambientales que afectarían la habitabilidad del planeta.

A pesar de estas advertencias de la ciencia, las tendencias señalan un aumento superior de temperatura que podría ubicarse alrededor de $4^{\circ} \mathrm{C}$, con lo cual, como lo dice un informe del Banco Mundial, "avanzamos hacia un incremento de $4^{\circ} \mathrm{C}$ de la temperatura del planeta [antes de que termine el siglo], lo cual provocará olas de calor extremo, disminución de las existencias de alimentos a nivel mundial, pérdida de 
ecosistemas y biodiversidad, y una elevación potencialmente mortal del nivel de los océanos" y agrega que "no hay además, seguridad alguna de que sea posible la adaptación a un mundo $4^{\circ} \mathrm{C}$ más cálido" ${ }^{2}$. Paradójicamente, a pesar de las múltiples reuniones internacionales para abordar este problema global, que vienen realizándose desde hace décadas, no se han producido nunca acuerdos vinculantes entre los países, en especial los Estados Unidos, la China y varios países emergentes, para limitar las emisiones de GEI. Esta falta de acción por parte de los gobiernos para abordar el mayor problema de la especie humana se debe en muy buena medida a la dificultad de cambiar el insostenible modelo económico actual; el capitalismo global desregulado.

\section{LA INGENIERÍA Y LA SOSTENIBILIDAD}

En la medida en que se ha evidenciado que la magnitud, la intensidad y la forma de los efectos de las actividades humanas han sobrepasado las capacidades de regeneración y restauración y la resiliencia de los ecosistemas y en general las del mundo natural, también se ha hecho claro que si queremos mantener las condiciones favorables para la vida, es necesario frenar las tendencias actuales de deterioro del ambiente planetario causadas por el Cambio Global y muy especialmente por el cambio climático, lo que nos obliga a realizar un cambio cultural y tecnológico profundo, para contribuir a construir un mundo sostenible, mejor ecológica y socialmente.

Desde la perspectiva de la ingeniería se han buscado maneras para lograr este objetivo a través de la reducción de los impactos ambientales y de la disminución del consumo de energía y de recursos naturales, aplicando nuevas formas de aprovechamiento y de producción de infraestructuras, bienes y servicios y desarrollando nuevas tecnologías ecoamigables.

Para abordar específicamente el problema descrito surgió un nuevo campo de la ingeniería, el de la Ingeniería Ambiental, que se derivó de la Ingeniería Civil. Con ella se ha buscado identificar, prevenir y mitigar los impactos de las diversas actividades sobre el medio ambiente y generar desarrollos tecnológicos ecoeficientes para reducir el consumo de energía y recursos naturales.

En los años sesenta del siglo pasado se propuso establecer un puente entre la ingeniería y la ecología, buscando satisfacer necesidades de la sociedad y de la naturaleza, que se denominó Ingeniería Ecológica (Ecological Engineering). Este concepto ha evolucionado y se ha centrado en la aplicación de las técnicas y herramientas de la ingeniería para analizar sistemas complejos, diseñando ecosistemas artificiales orientados al tratamiento de aguas residuales y la restauración de ecosistemas degradados.

La inquietud por el cuidado del ambiente y sus recursos se ha extendido a muchas otras ramas de la ingeniería y hoy en día el ahorro de energía y la ecoeficiencia son variables muy importantes en el diseño de nuevos equipos y productos, como motores, equipos de transporte, desarrollo de fuentes de energía limpias y sistemas de tratamiento de aguas residuales para citar algunos ejemplos. Las buenas prácticas para conservar el ambiente y reducir el consumo de recursos naturales también se han convertido en estándares de la industria.

La preocupación por la salud y la sostenibilidad del planeta ha influenciado otras disciplinas. Así por ejemplo, buscando mantener el sistema económico, pero tratando de encontrar el necesario equilibrio entre desarrollo y ambiente, se han generado nuevas ideas, conceptos y productos, que buscan disminuir el impacto ambiental y el consumo de energía y recursos naturales. La versión actual de esta nueva visión se ha bautizado como Economía Ver$\mathrm{de}^{2} \mathrm{y}$, ha sido adoptada con entusiasmo por muchos gobiernos y organizaciones, entre ellas las Naciones Unidas, con la esperanza de que su aplicación, liderada fundamentalmente por el sector privado, sea suficiente para lograr el cambio de las tendencias que dibujan un futuro incierto y potencialmente catastrófico, sin tener que recurrir a tomar medidas más radicales que puedan interferir con el avance del modelo del capitalismo globalizado no regulado.

Se está hablando también de un nuevo concepto, el de la Economía Circular, que a partir de una perspectiva ecológica, cambia el concepto de la economía tradicional que considera el sistema económico como el centro de un sistema abierto en el cual los recursos naturales que entran se consideran ilimitados, al igual que la capacidad de la naturaleza para recibir y procesar los residuos y deshechos del sistema económico. La diferencia esencial entre ellos consiste en entender el sistema económico como una de las múltiples partes de la ecosfera, que se concibe como un sistema cerrado y limitado, cuyo funcionamiento está impulsado por la energía que recibe del sol y que opera sobre la base de ciclos que en muy buena medida incorporan los residuos como lo hace el mundo natural. La Figura 3 presenta de forma simplificada estos conceptos.

A pesar de los nuevos planteamientos y de los esfuerzos y los logros tecnológicos realizados hasta ahora, la

\footnotetext{
${ }^{1}$ Citado por Kolbert en La Sexta Extinción- Una historia nada natural.

${ }^{2}$ Definida por el PNUMA como el "mejoramiento del bienestar humano e igualdad social, mientras que se reduce significativamente los riesgos medioambientales y la escasez ecológica”.
} 


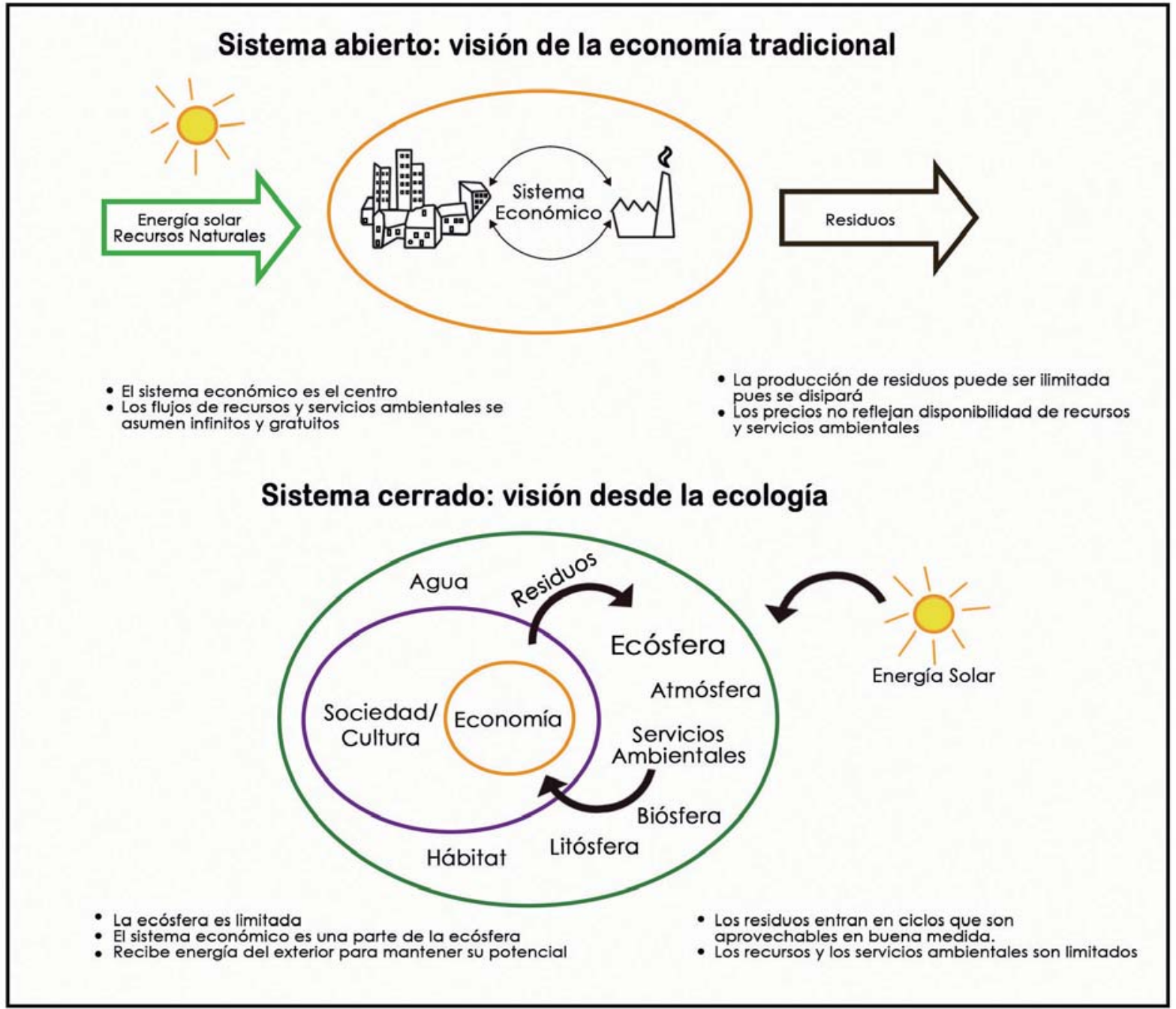

Figura 3. Comparación de las visiones para lograr la sostenibilidad.

Fuente: Elaboración propia.

dura realidad que presentan los indicadores de calentamiento y deterioro de la salud del planeta y sus ecosistemas, es que su tendencia de crecimiento se mantiene. Por tanto, la gran duda que subsiste en un grupo grande de los científicos del clima, de ambientalistas y de buena parte de la sociedad es si Economía Verde y las nuevas tecnologías son suficientes para reversar estas tendencias. Además, las proyecciones indican que el escaso tiempo de que aún disponemos para evitar la posibilidad de entrar en procesos de cambios no lineales e irreversibles de pérdida de la calidad ambiental del planeta, exige medidas más contundentes y radicales.

Desde el punto de vista conceptual es necesario señalar que la relación entre la sociedad y la naturaleza ha estado dominada históricamente, al menos en la cultura occidental, por el antropocentrismo, que ubica al ser humano como dueño y beneficiario del mundo natural y de los bienes y servicios que éste genera, lo cual nos ha conducido a la peligrosa situación actual. En contraposición, hoy en día se está buscando una nueva relación entre la sociedad y la naturaleza basada en el biocentrismo, en la que el ser humano, homo sapiens, deja de ser el centro del mundo, para convertirse en una de las muchas especies que compartimos el planeta y que para lograr la sostenibilidad del mismo es necesario conocer y respetar a todos los seres y sus derechos. La aproximación biocéntrica implica una nueva relación más justa y ética, entre la sociedad y la naturaleza, basada en un enfoque de derechos y en la comprensión de la integralidad y las limitaciones del mundo natural. Por lo tanto, la relación sociedad-naturaleza y el tratamiento de la problemática ambiental dejan de ser 
asuntos exclusivamente técnicos, para incorporar conjuntamente variables y dimensiones éticas, económicas y sociales, lo que los transforma en temas multivariados y complejos.

La propuesta que hace este artículo desde la perspectiva de la evolución de la ingeniería y siguiendo tendencias internacionales, es la de crear una nueva ingeniería; la Ingeniería de Ecosistemas, que no debe entenderse como una más de las especializaciones de la ingeniería, sino como un cambio en la concepción tradicional de la ingeniería centrada en la explotación y la transformación de los recursos naturales no renovables y por tanto limitados, hacia otra centrada en la conservación y el aprovechamiento sostenible de los recursos renovables, para ser utilizados como fuentes de energías limpias y de materias primas para toda clase de procesos de transformación y desarrollo incluyendo nuevos materiales y productos.

Esta propuesta no es para nada incompatible con las ingenierías tradicionales. Por el contrario, su propósito es articularse con ellas para complementarlas introduciendo el conocimiento del mundo natural; de sus características, funcionamiento y limitaciones, para la concepción, enseñanza y ejercicio de la profesión. Así pues, la tarea de los ingenieros de ecosistemas, sería la de apoyar el trabajo que hace la naturaleza para generar los bienes y servicios que nos brindan los ecosistemas, con los métodos, capacidades y herramientas de la ingeniería.

La diferencia fundamental entre las dos aproximaciones descritas radica en que lo que buscan los avances que se han logrado es disminuir el consumo de energía y de recursos naturales en las diversas actividades y procesos, mitigar sus impactos ambientales o restaurar ecosistemas degradados. En una palabra, disminuir la huella de las actividades humanas. El aporte de la Ingeniería de Ecosistemas que se propone en este artículo es complementar los valiosos esfuerzos realizados con una nueva ingeniería, cuyo propósito principal es lograr el aumento de la resiliencia y de la resistencia de los ecosistemas para enfrentar los cambios causados por las actividades humanas y mantener, e incluso incrementar, la capacidad de generación de bienes y servicios ecosistémicos para responder a las presiones que crea el nuevo escenario que nos ofrece el Antropoceno.

De manera simplificada podría decirse que la propuesta consiste en el paso de una ingeniería basada en el aprovechamiento del mundo inanimado, a una basada en el del mundo biótico y los ecosistemas, cuyo objetivo es la búsqueda de la sostenibilidad.

Un propósito fundamental de la Ingeniería de Ecosistemas es apoyar a la naturaleza para lograr la conservación e incluso el aumento de la capacidad de los ecosistemas para generar sus bienes y servicios. Las tendencias actuales de destrucción de los ecosistemas naturales para dedicar el espacio que ocupan a otros usos pone en riesgo la disponibilidad de estos bienes y servicios vitales y crea nuevas situaciones y problemas imprevisibles, como los causados por la desaparición de las abejas y otros polinizadores por la contaminación electromagnética de origen humano, o la de los bosques tropicales y sus efectos reguladores sobre el clima, o la desaparición de los corales esenciales para la vida marina.

La Evaluación de Ecosistemas del Milenio publicada por las Naciones Unidas en 2005, después de un trabajo de cuatro años en el que participaron más de 1300 científicos de todo el mundo, puso de presente el deterioro de los ecosistemas, formalizó este tema, definió sus servicios como los recursos y procesos generados por los ecosistemas naturales que hacen posible el funcionamiento de la naturaleza y benefician a las personas y a la sociedad, al suministrarles bienes y servicios esenciales para la vida y el progreso social. También clasificó los servicios ecosistémicos en cuatro categorías; de aprovisionamiento, como la producción de agua, de alimentos y de materias primas vegetales; de regulación, como el control del clima, de las catástrofes como las inundaciones y de las enfermedades; de apoyo, como los ciclos de nutrientes y de deshechos y la polinización; y culturales como beneficios espirituales, estéticos y recreativos.

La Figura 4 presenta estas categorías y sus relaciones e importancia para el bienestar humano y para la sociedad.

Sintetizando la propuesta que se hace en este artículo, puede decirse que el objeto de la Ingeniería de Ecosistemas es estudiar, comprender y actuar sobre los recursos renovables, para apoyar el trabajo de la naturaleza, aprovechando las capacidades y posibilidades que la ingeniería ofrece. Es decir que busca la conservación y aprovechamiento sostenible de los ecosistemas, investigando sobre su funcionamiento y aplicando las herramientas y técnicas para lograr que la generación de los bienes y los servicios ecosistémicos esenciales para la vida y el progreso se mantengan a lo largo del tiempo y contribuyan a la sostenibilidad del planeta.

La Ingeniería de Ecosistemas modifica la tendencia al fraccionamiento y la especialización exagerada que ha seguido la ingeniería tradicional, al requerir un enfoque complejo e integrador dada la naturaleza compleja, relacional, interdisciplinaria e interactiva de su objeto de estudio y trabajo. La propuesta de una ingeniería de ecosistemas implica además, los aportes de las ciencias sociales y naturales, de la economía, del derecho y de muchas otras disciplinas, al requerir para el logro de sus objetivos un enfoque complejo e integrador que responda a la naturaleza multivariada, relacional e interactiva del mundo natural.

Por último, es indispensable destacar que el éxito de la propuesta que se describe aquí depende de que su aplicación se enmarque en un conjunto de principios y de valores de categoría superior, que están implícitos en el concepto de la sostenibilidad, como son la comprensión de que formamos parte de un todo interactivo, el respeto por 


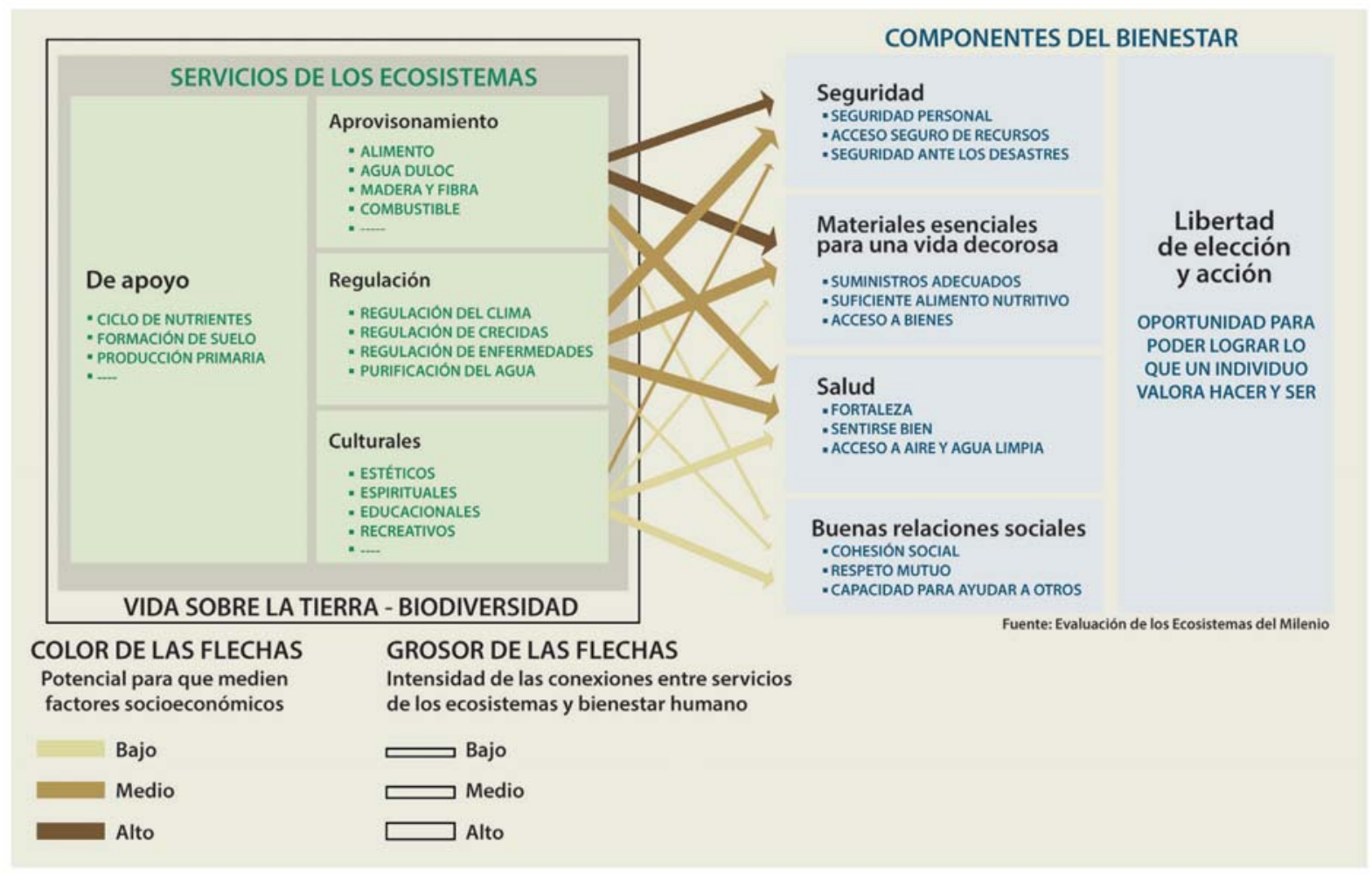

Figura 4. Clasificación de los servicios de los ecosistemas según la Evaluación de Ecosistemas del Milenio.

Fuente: PNUMA, Evaluación de los Ecosistemas del Milenio.

el mundo maravilloso y único que nos ha alojado tan generosamente a todos los seres con quienes lo compartimos y la equidad entre los habitantes actuales del planeta y con las generaciones futuras.

\section{REFERENCIAS}

Carrizosa, J. (2006). Desequilibrios territoriales y sostenibilidad local. Bogotá: Universidad Nacional de Colombia, Sede Bogotá, Instituto de Estudios Ambientales, IDEA.
Guhl Nannetti, E. (2013). La región hídrica de Cundinamarca-Bogotá, una propuesta conceptual: Articulación conceptual de la gestión integrada del agua, el ordenamiento territorial y la sostenibilidad. Bogotá: Empresa de Acueducto y Alcantarillado de Bogotá (EAB).

Guhl Nannetti, E. (2015). Lecturas Ambientales \# 2 y 3. Manuscrito inédito.

Kolbert, E. (2015). La Sexta Extinción. Una historia nada natural. Barcelona: Crítica.
Klein, N. 2015. Esto lo cambia todo. El capitalismo contra el clima. Bogotá: Editorial Planeta Colombiana.

Vogt, K., Gordon, J.C., Wargo, et al. (1997). Ecosystems: Balancing Science with Management. New York: Springer.

World Resources Institute (2005). Ecosystems Millennium Assessment: Ecosystems and Human Well-being. Washington: WRI. 